\title{
Apoptosis-Inducing Factor: Mechanistic Insights and Therapeutic Opportunities from a Metabolic, Allosteric Switch
}

\author{
Chris A. Brosey ${ }^{a}$, Runze Shen ${ }^{b}$, Kathryn Burnettc ${ }^{c}$ Greg Hura $^{d}$, and John A. Tainer \\ aMolecular \& Cellular Oncology, M.D. Anderson Cancer Center, Houston, TX, 77030, USA, \\ CABrosey@mdanderson.org \\ ${ }^{b}$ Molecular \& Cellular Oncology, M.D. Anderson Cancer Center, Houston, TX, 77030, USA, \\ RShen3@mdanderson.org \\ cMolecular Biophysics and Integrated Bioimaging Division (MBIB), Lawrence Berkeley National \\ Laboratory, Berkeley, CA 94720, USA, kburnett@lbl.gov \\ ${ }^{d}$ Molecular Biophysics and Integrated Bioimaging Division (MBIB), Lawrence Berkeley National \\ Laboratory, Berkeley, CA 94720, USA | Chemistry \& Biochemistry, University of California Santa \\ Cruz, Santa Cruz, CA 95064, USA, ghura@lbl.gov \\ eMolecular and Cellular Oncology and Cancer Biology, M.D. Anderson Cancer Center, Houston, \\ TX, 77030, USA | Molecular Biology Consortium, Lawrence Berkeley National Laboratory, \\ Berkeley, CA 94720, USA JTainer@mdanderson.org
}

Mitochondrial-nuclear crosstalk is increasingly recognized as critical to cellular health and metabolic homeostasis. An understudied regulator of cell fate, mitochondrial Apoptosis-Inducing Factor (AIF) partners with disulfide relay chaperone Mia40/CHCHD4 of the IMS import pathway to promote respiratory complex biogenesis [1,2]. Following excessive DNA damage, AIF functions as a mitochondrial sensor for nuclear poly(ADP-ribose) DNA damage signaling, initiating caspase-independent cell death (parthanatos) upon irrevocable genomic injury and $\mathrm{NAD}^{+}$ depletion. AlF's dual roles in oxidative phosphorylation and cell fate are attractive therapeutic targets for combination treatments in cancer. However, the molecular mechanisms regulating AIF functions and its conversion from mitochondrial to nuclear death factor are poorly understood.

Binding to NADH and formation of a long-lived charge-transfer complex (CTC) allosterically triggers architectural switching of AIF from monomer to dimer and enables interaction with disulfide relay chaperone Mia40. By integrating data from small-angle X-ray scattering (SAXS), X-ray crystallography, and computation, we have previously defined the molecular mechanisms linking AIF's central NADH active site to its dimerization surface [3]. We have now uncovered how $\mathrm{NADH}$-driven allosteric switching regulates AIF's role in mitochondrial import with a crystallized complex of AIF and the N-terminal Mia40 domain. This structure reveals a novel interaction surface across AlF's C-terminal domain and highlights how NADH allosterically controls the interface through a buried aromatic relay to enable Mia40 binding. Testing of the AIF binding surface demonstrates that contact residues also enable the long-lived stability of AIF-CTC complexes. These results provide the first molecular explanation for how AIF connects mitochondrial import to metabolic state and are expected to illuminate how AIF integrates NAD $(H)$ sensing to link mitochondrial homeostasis, DNA damage signaling, and cell death.

References

[1] Hangen et al. (2015) Molecular Cell, 58:1001-14 (2015).

[2] Meyer et al. (2015) Cell Death and Disease, 6:e1814 (2015).

[3] Brosey et al., (2016) Structure, 24:2067-2079 (2016). 